\title{
Dolor y variabilidad de la frecuencia cardíaca en recién nacidos prematuros que recibieron surfactante: un estudio piloto
}

\author{
Neonatal pain and heart rate variability in preterm infants treated with surfactant: \\ a pilot study
}

Dra. Nilufer Okur ${ }^{a}$, Dra. Nurdan Uras ${ }^{a}$, Dr. Mehmet Buyuktiryaki ${ }^{a}$, Dr. Mehmet Y. Oncel ${ }^{b}$,

Dra. Fatma N. Sari ${ }^{a}$ Dr. Erbu Yarcia, Dra. Evrim A. Dizdar ${ }^{a}$, Dr. Fuat E. Canpolat y Dra. Serife S. Oguz ${ }^{a}$

\begin{abstract}
RESUMEN
Objetivo. Evaluar la percepción del dolor de recién nacidos prematuros a quienes se les administró surfactante mediante diferentes técnicas, utilizando la variabilidad de la frecuencia cardíaca (VFC).

Métodos. Se aleatorizó a los recién nacidos que requirieron tratamiento con surfactante por SDR a los grupos INSURE o MIST. El análisis de la VFC se realizó con la tecnología NIPE para evaluar el componente parasimpático del sistema nervioso autónomo de los recién nacidos. Se registró la VFC antes, durante y después de administrar el surfactante. La evaluación del dolor se determinó con la escala PIPP.

Resultados. Se incluyó a 14 recién nacidos en el estudio. Los grupos tenían características demográficas similares. Los puntajes de la escala PIPP no difirieron entre los grupos INSURE y MIST $(p=0,05)$. Se observó una diferencia estadísticamente significativa en la mediana de la VFC durante la administración del surfactante entre los grupos INSURE y MIST (52 frente a $56, p=0,03)$. El análisis de la VFC fue similar entre los grupos antes y después de administrar el surfactante.

Conclusión. La administración de surfactante mediante la técnica MIST podría ser más cómoda para los recién nacidos prematuros con SDR. No obstante, es necesario realizar otros estudios con series más importantes.

Palabras clave: intubación, recién nacido prematuro, dolor, surfactante pulmonar.
\end{abstract}

http: / / dx.doi.org/10.5546/ aap.2019.397

Texto completo en inglés:

http: / / dx.doi.org/10.5546/ aap.2019.eng.397

a. División de Neonatología. Hospital Universitario Maternal Zekai Tahir Burak, Ankara, Turquía.

b. Kâtip Çelebi Üniversitesi, Facultad de Medicina, Unidad de Cuidados Intensivos Neonatales, Esmirna, Turquía.

Correspondencia:

Dra. Nilufer Okur: n.matur@hotmail.com

Financiaciamiento: Ninguno.

Conflicto de intereses: Ninguno que declarar.

Recibido: 9-7-2018

Aceptado: 27-6-2019
Cómo citar: Okur N, Uras N, Buyuktiryaki M, Oncel MY, et al. Dolor y variabilidad de la frecuencia cardíaca en recién nacidos prematuros que recibieron surfactante: un estudio piloto. Arch Argent Pediatr 2019;117(6):397-401.

\section{INTRODUCCIÓN}

El surfactante se aplica mediante dos técnicas diferentes para tratar el síndrome de dificultad respiratoria (SDR). El método INSURE (intubación, surfactante, extubación) es el más frecuente para el tratamiento con surfactante. ${ }^{1}$ En el último tiempo, se desarrolló un nuevo método para administrar surfactante: el tratamiento con surfactante mediante una técnica mínimamente invasiva (minimally invasive surfactant therapy, MIST).

Esta técnica se usa clínicamente para la administración de surfactante a través de un catéter endotraqueal de pequeño calibre durante la respiración espontánea con presión positiva continua en las vías respiratorias (continuous positive airway pressure, CPAP). ${ }^{2-5}$ Sin embargo, no hay suficientes datos o experiencia respecto de cómo las técnicas MIST e INSURE afectan la percepción del dolor en los recién nacidos.

Es difícil evaluar la percepción del dolor en los lactantes. En la actualidad, la evaluación del dolor o malestar se basa principalmente en escalas conductuales del dolor. No obstante, estos sistemas de puntaje tienen en cuenta ítems como la expresión facial, los movimientos corporales o la capacidad de confort, por lo que están sujetos a una variabilidad inter e intraobservador significativa. ${ }^{6-9}$

En estudios recientes, se indicó que las influencias simpáticas y parasimpáticas en respuesta a estímulos de dolor o estrés pueden medirse mediante variaciones de los intervalos RR de la frecuencia cardíaca. Se demostró que el índice que evalúa el componente 
parasimpático en los recién nacidos (newborn infant parasympathetic evaluation, NIPE) podría evaluar el tono parasimpático y dolor prolongado, dolor agudo, malestar y estímulos agradables. En este estudio, se intentó comparar la percepción del dolor de recién nacidos prematuros a los que se les administró surfactante mediante diferentes técnicas, usando la variabilidad de la frecuencia cardíaca (VFC) medida con el índice NIPE y el puntaje PIPP (premature infant pain profile).

\section{MÉTODOS}

Este estudio controlado aleatorizado y prospectivo se llevó a cabo en la unidad de cuidados intensivos neonatales (UCIN) del Hospital Universitario Maternal Zekai Tahir Burak entre mayo y junio de 2015. Se incluyó a recién nacidos prematuros con una edad gestacional de entre 26 y 32 semanas y un peso al nacer $\leq 1250 \mathrm{~g}$ que tuvieron SDR. Este estudio fue aprobado por el Comité de Ética del Hospital Universitario Maternal Zekai Tahir Burak. Se obtuvo el consentimiento por escrito de los padres de cada recién nacido.
Se aleatorizó a los recién nacidos que requerían tratamiento con surfactante por SDR a los grupos INSURE o MIST cuando ingresaron en la UCIN. A cada recién nacido se le colocó el monitor NIPE (Mdoloris Medical Systems, Francia) antes de administrarle el surfactante. Se excluyó a los recién nacidos con anomalías congénitas importantes, asistencia respiratoria con presión positiva (ARPP) o intubación en la sala de partos, anomalías cardíacas estructurales, exposición a procedimientos dolorosos como venopunción, aspiración, obtención de muestras de sangre o pinchazo en el talón 30 minutos antes de la evaluación, y falta de consentimiento de los padres. Se diagnosticó SDR a los recién nacidos que tenían los siguientes síntomas: necesidad de oxigenoterapia, taquipnea, quejidos espiratorios y tiraje intercostal. Se confirmó el diagnóstico mediante hallazgos radiológicos y gasométricos clásicos. Un protocolo sugerido sería tratar a los recién nacidos de $\leq 26$ semanas de gestación cuando la demanda de $\mathrm{FiO}_{2}$ es $>0,30$ y a los recién nacidos de más de 26 semanas de gestación cuando la demanda de $\mathrm{FiO}_{2}$ es $>0,40$.

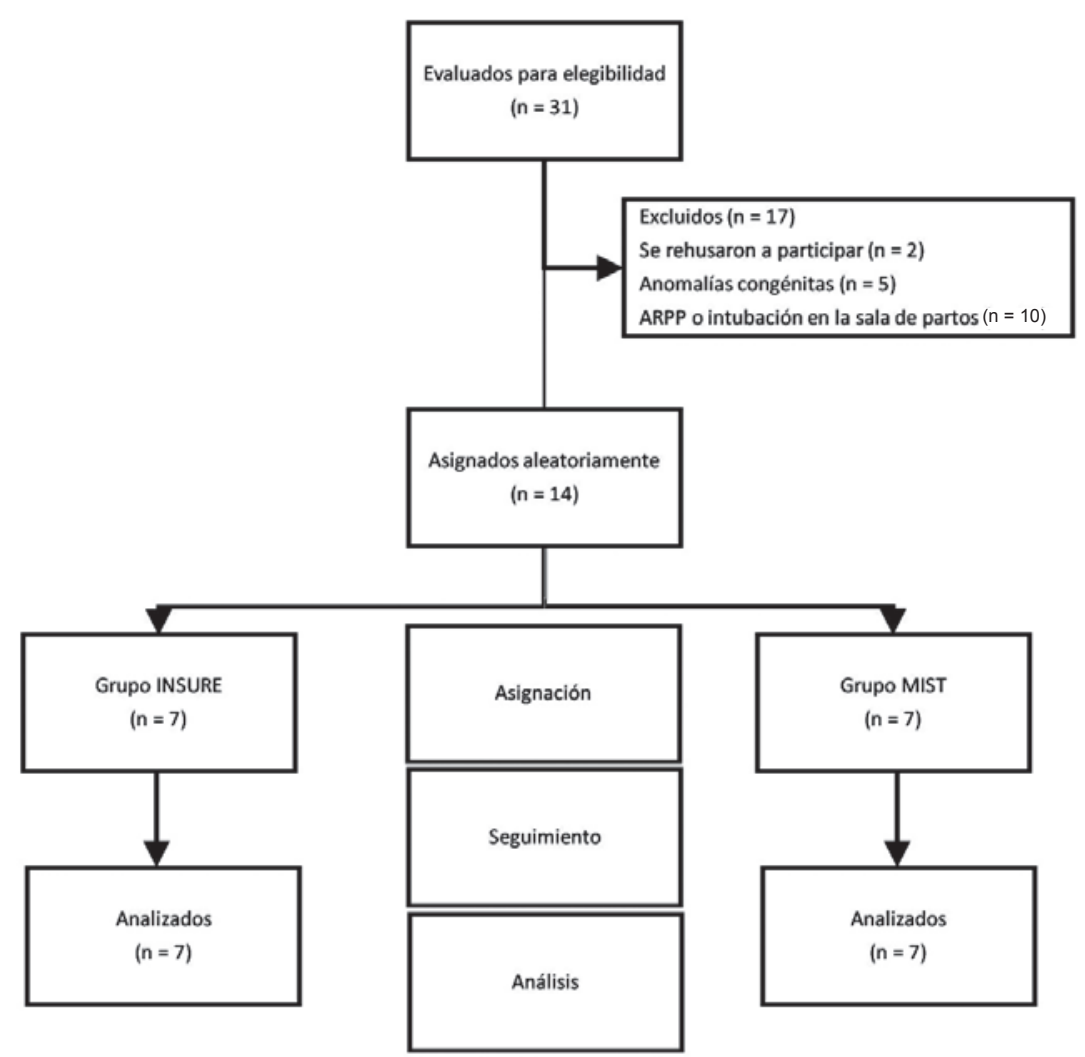

ARPP: asistencia respiratoria con presión positiva. 


\section{Estrategias de tratamiento}

Se aplicó el tratamiento con surfactante con el procedimiento MIST a los recién nacidos usando una sonda nasogástrica estéril y flexible de 5 F. Se instiló surfactante en bolo durante 30 a 60 segundos y se retiró el catéter traqueal de inmediato.

A los recién nacidos que recibieron surfactante con la técnica INSURE primero se los intubó por vía oral con un tubo endotraqueal con doble luz y se les instiló surfactante en la tráquea durante 30 segundos. Se insuflaron los pulmones de forma manual con un dispositivo con pieza en $\mathrm{T}$ a una presión de $20 / 5 \mathrm{cmH}_{2} \mathrm{O}$ durante la instilación de surfactante, y luego se extubó al recién nacido de inmediato.

Se administró poractante alfa (Curosurf; Chiesi Farmaceutici, Parma, Italia) a todos los recién nacidos (200 mg/ kg/dosis para cada paciente) del estudio. Se registró el índice NIPE de cada recién nacido de forma consecutiva, antes, durante y después de la administración del surfactante. Se utilizó la escala PIPP para medir las respuestas conductuales y fisiológicas de los recién nacidos al dolor. ${ }^{10}$

\section{Análisis estadístico}

Para los análisis estadísticos, se utilizó el paquete estadístico SPSS para Windows v.17.0 (SPSS Inc., Chicago, Illinois). Los datos con distribución normal se expresaron como

TABLA 1. Características demográficas de los grupos

\begin{tabular}{lccc}
\hline & $\begin{array}{c}\text { INSURE } \\
(\mathbf{n}=7)\end{array}$ & $\begin{array}{c}\text { MIST } \\
(\mathbf{n}=7)\end{array}$ & $p$ \\
\hline Edad gestacional, semanas $^{\mathrm{a}}$ & $29,1 \pm 1,5$ & $29,4 \pm 1,2$ & 0,71 \\
Peso al nacer, ga $^{\text {a }}$ & $1107 \pm 103$ & $1127 \pm 85$ & 0,69 \\
Sexo (masculino), n & 4 & 3 & 0,50 \\
Cesárea, n & 5 & 6 & 0,50 \\
Uso prenatal de corticoesteroides, $\mathrm{n}$ & 7 & 5 & 0,23 \\
\hline${ }^{a}$ media \pm desviación estándar. & & &
\end{tabular}

media y desviación estándar; los demás, como mediana e intervalo. Se compararon el porcentaje demográfico y las medias de las variables de los pacientes entre los dos grupos con la prueba exacta de Fisher, la prueba $\chi^{2}$ y la prueba $t$. Un valor de $p<0,05$ se consideró significativo en términos estadísticos.

Se calculó el tamaño de la muestra con datos provenientes de los registros de la UCIN relativos al período previo al estudio. Los recién nacidos que habían sido tratados con surfactante tenían un índice NIPE de 56 (media geométrica, distribución normal logarítmica) con una desviación estándar de 9 durante el período previo al estudio. Para lograr una reducción del $30 \%$ en el índice NIPE con una prueba de dos colas con un error de tipo alfa de 0,05 y un error de tipo beta de 0,2, se debió aleatorizar a 14 recién nacidos.

\section{RESULTADOS}

Se incluyó a 14 recién nacidos en el estudio: INSURE: $\mathrm{n}=7$; MIST: $\mathrm{n}=7$ (Figura 1). La edad gestacional media $( \pm \mathrm{DE})$ de los recién nacidos fue de 29,1 $\pm 1,5$ en el grupo INSURE y 29,4 \pm 1 ,2 en el grupo MIST $(p>0,05)$. La media del peso al nacer ( \pm DE) de los recién nacidos fue $1107 \pm 103$ g en el grupo INSURE y $1127 \pm 85 \mathrm{~g}$ en el grupo MIST $(p>0,05)$. Las características demográficas de los grupos se describen en la Tabla 1.

Los puntajes PIPP y las variables de la VFC de los grupos se presentan en la Tabla 2. La mediana de los puntajes PIPP fue 10 (10-13) en el grupo INSURE y 10 (8-12) en el grupo MIST. Los puntajes PIPP no difirieron entre los recién nacidos de los grupos INSURE y MIST $(p=0,053)$. Se observó una diferencia estadísticamente significativa en la mediana de la VFC de los recién nacidos durante la administración del surfactante entre los grupos INSURE y MIST (52 frente a 56, $p=0,03$ ). Los análisis de la VFC antes y después de la administración del surfactante fueron similares en ambos grupos (Tabla 2). En la

TABLA 2. Comparación del índice de VFC y los puntajes PIPP

\begin{tabular}{|c|c|c|c|}
\hline & $\begin{array}{c}\text { INSURE } \\
(\mathrm{n}=7)\end{array}$ & $\begin{array}{l}\text { MIST } \\
(n=7)\end{array}$ & $p$ \\
\hline Índice de VFC, antes del tratamiento con surfactante & $64(53-68)$ & $62(58-80)$ & 0,805 \\
\hline Índice de VFC, durante el tratamiento con surfactante & $52(50-55)$ & $56(50-75)$ & 0,038 \\
\hline Índice de VFC, 20 min después del tratamiento con surfactante & $56(54-60)$ & $61(52-80)$ & 0,053 \\
\hline Índice de VFC, 40 min después del tratamiento con surfactante & $60(50-69)$ & $61(54-82)$ & 0,456 \\
\hline Índice de VFC, 60 min después del tratamiento con surfactante & $60(53-75)$ & $71(52-89)$ & 0,383 \\
\hline Puntajes PIPP durante el tratamiento con surfactante & $10(10-13)$ & $10(8-12)$ & 0,053 \\
\hline
\end{tabular}

Mediana (mínimo-máximo). VFC: variabilidad de la frecuencia cardíaca. PIPP: perfil de dolor del prematuro. 
TABLA 3. Índice NIPE y puntaje PIPP de cada recién nacido

\begin{tabular}{|c|c|c|c|c|c|c|c|c|c|}
\hline Caso & SG & PN & $\begin{array}{c}\text { Técnica de } \\
\text { administración } \\
\text { de surfactante }\end{array}$ & $\begin{array}{c}\text { Índice NIPE } \\
\text { Antes del } \\
\text { tratamiento } \\
\text { con surfactante }\end{array}$ & $\begin{array}{c}\text { Índice NIPE } \\
\text { Durante el } \\
\text { tratamiento } \\
\text { con surfactante }\end{array}$ & $\begin{array}{c}\text { Índice NIPE } \\
20 \text { min después } \\
\text { tratamiento } \\
\text { con surfactante }\end{array}$ & $\begin{array}{c}\text { Índice NIPE } \\
40 \text { min después } \\
\text { tratamiento } \\
\text { con surfactante }\end{array}$ & $\begin{array}{l}\text { Índice NIPE } \\
60 \text { min después } \\
\text { tratamiento } \\
\text { con surfactante }\end{array}$ & $\begin{array}{l}\text { Puntaje } \\
\text { PIPP }\end{array}$ \\
\hline 1 & 30 & 1150 & MIST & 62,00 & 56,00 & 61,00 & 57,00 & 52,00 & 10,00 \\
\hline 2 & 27 & 980 & MIST & 58,00 & 54,00 & 62,00 & 72,00 & 71,00 & 11,00 \\
\hline 3 & 29 & 1160 & MIST & 62,00 & 54,00 & 60,00 & 61,00 & 62,00 & 10,00 \\
\hline 4 & 31 & 1250 & MIST & 80,00 & 75,00 & 80,00 & 82,00 & 89,00 & 8,00 \\
\hline 5 & 30 & 1120 & MIST & 70,00 & 72,00 & 73,00 & 72,00 & 75,00 & 9,00 \\
\hline 6 & 30 & 1170 & MIST & 58,00 & 50,00 & 52,00 & 54,00 & 54,00 & 12,00 \\
\hline 7 & 29 & 1065 & MIST & 60,00 & 58,00 & 58,00 & 58,00 & 84,00 & 10,00 \\
\hline 8 & 29 & 1020 & INSURE & 53,00 & 50,00 & 56,00 & 50,00 & 53,00 & 12,00 \\
\hline 9 & 30 & 1200 & INSURE & 57,00 & 55,00 & 60,00 & 67,00 & 60,00 & 11,00 \\
\hline 10 & 28 & 1050 & INSURE & 60,00 & 53,00 & 56,00 & 58,00 & 55,00 & 12,00 \\
\hline 11 & 31 & 1220 & INSURE & 68,00 & 52,00 & 58,00 & 69,00 & 75,00 & 10,00 \\
\hline 12 & 31 & 1230 & INSURE & 64,00 & 52,00 & 56,00 & 60,00 & 64,00 & 11,00 \\
\hline 13 & 28 & 1030 & INSURE & 65,00 & 53,00 & 58,00 & 62,00 & 63,00 & 11,00 \\
\hline 14 & 27 & 1000 & INSURE & 66,00 & 51,00 & 54,00 & 54,00 & 55,00 & 13,00 \\
\hline
\end{tabular}

Tabla 3, se presentan el índice de VFC y el puntaje PIPP de cada recién nacido. Se observó una correlación significativa entre los valores de NIPE y los puntajes PIPP durante la administración del surfactante $\left(\mathrm{r}^{2}=-0,811, p<0,0001\right)$.

\section{DISCUSIÓN}

En este estudio, se observó que el índice NIPE era significativamente más alto durante la administración del surfactante en los recién nacidos tratados con la técnica MIST y también se correlacionó con los puntajes PIPP.

Se ha llegado al consenso de que la intubación endotraqueal debe realizarse cuando el recién nacido está sedado correctamente. ${ }^{10,11}$ No obstante, actualmente se debate si debe utilizarse la sedación durante la técnica MIST, dado que la respiración espontánea es una condición previa para este procedimiento. En los estudios donde se evaluó la técnica MIST, no se utilizó sedación. ${ }^{12}$

En nuestro estudio, se observó que el grupo MIST sintió menos dolor que el grupo INSURE. Por lo tanto, sugerimos que la administración de surfactante mediante la técnica MIST podría ser más cómoda para los recién nacidos prematuros con SDR.

Alexandre y cols., compararon la VFC antes y después de las fases estandarizadas de cocooning (rodear al recién nacido con los brazos de manera protectora) asociadas con la voz humana y realizadas: 1) por la madre y 2) por un tercero. Se evaluó la VFC y se la expresó como un índice que refleja el tono parasimpático. Se demostró que el cocooning asociado con la voz humana mejora la VFC en los recién nacidos prematuros, con un aumento de la actividad parasimpática. ${ }^{13}$

Nuestro estudio demostró ser sólido a la hora de revelar los niveles de estrés de los recién nacidos prematuros tratados con surfactante. Se midió el dolor con los puntajes PIPP y el índice NIPE y se utilizaron diferentes técnicas para administrarles surfactante a los recién nacidos. El puntaje PIPP es una escala importante que se ha utilizado para evaluar de forma objetiva el dolor en los recién nacidos prematuros.

En la bibliografía, hay poca información sobre la sensación de dolor que puede provocar la técnica MIST, y en nuestro estudio, se presentan resultados que ayudarán a clarificar esta área de investigación. Además, este estudio es el primero en comparar el puntaje PIPP y el índice NIPE en recién nacidos prematuros.

Nuestro estudio presenta ciertas limitaciones. En primer lugar, la VFC está controlada por el flujo de salida del sistema nervioso autónomo, que puede verse alterado por diferentes factores, que incluyen la maduración, las condiciones ambientales o los fármacos.

En segundo lugar, se trata de un estudio con una muestra pequeña. 


\section{CONCLUSIÓN}

Nuestros resultados sugieren que la administración de surfactante mediante la técnica MIST podría ser más cómoda para los recién nacidos prematuros con SDR.

\section{REFERENCIAS}

1. Bohlin K, Gudmundsdottir T, Katz-Salamon M, Jonsson $\mathrm{B}$, Blennow M. Implementation of surfactant treatment during continuous positive airway pressure. J Perinatol. 2007; 27(7):422-7.

2. Göpel W, Kribs A, Ziegler A, Laux R, et al. Avoidance of mechanical ventilation by surfactant treatment of spontaneously breathing preterm infants (AMV): an open-label, randomised, controlled trial. Lancet. 2011; 378(9803):1627-34.

3. Kribs A, Vierzig A, Hünseler C, Eifinger F, et al. Early surfactant in spontaneously breathing with nCPAP in ELBW infants-a single centre of our year experience. Acta Paediatr. 2008; 97(3):293-8

4. Kribs A, Härtel C, Kattner E, Vochem M, et al. Surfactant without intubation in preterm infants with respiratory distress: first multi-center data. Klin Padiatr. 2010; 222(1):13-7.

5. Kribs A, Pillekamp F, Hünseler C, Vierzig A, Roth B. Early administration of surfactant in spontaneous breathing with nCPAP: feasibility and outcome in extremely premature infants (postmenstrual age $</=27$ weeks). Paediatr Anaesth . 2007; 17(4):364-9.
6. Grunau RE, Oberlander T, Holsti L, Whitfield MF. Bedside application of the Neonatal Facial Coding System in pain assessment of premature neonates. Pain. 1998; 76(3): 277-86.

7. Carbajal R, Paupe A, Hoenn E, Lenclen R, Olivier-Martin M. DAN: une échelle comportementale d'evaluation de la douleur aiguë du nouveauné. Arch Pediatr. 1997; 4(7):623-8.

8. Stevens B, Johnston C, Petryshen P, Taddio A. Premature Infant Pain Profile: development and initial validation. Clin J Pain. 1996; 12(1):13-22.

9. Logier R, De Jonckheere J, Delecroix M, Keribedj A, et al. Heart rate variability analysis for arterial hypertension etiological diagnosis during surgical procedures under tourniquet. Conf Proc IEEE Eng Med Biol Soc. 2011; 2011:3776-9.

10. Friesen RH, Honda AT, Thieme RE. Changes in anterior fontanel pressure in preterm neonates during tracheal intubation. Anesth Analg. 1987; 66(9):874-8.

11. Pokela ML, Koivisto M. Physiological changes, plasma betaendorphin and cortisol responses to tracheal intubation in neonates. Acta Paediatr. 1994; 83(2):151-6.

12. Dargaville PA, Aiyappan A, De Paoli AG, Kuschel CA, et al. Minimally-invasive surfactant therapy in preterm infants on continuous positive airway pressure. Arch Dis Child Fetal Neonatal Ed. 2013; 98(2):F122-6.

13. Alexandre C, De Jonckheere J, Rakza T, Mur S, et al. Impact du cocooning et de la voix humaine sur le systéme nerveux autonome (SNA) del'enfant grand prématuré. Arch Pediatr. 2013; 20(9):963-8.

\title{
Salud respiratoria y del sueño infantil tras una amniocentesis en el segundo trimestre Child's respiratory and sleep health following mid-trimester amniocentesis
}

\author{
Prof. Dr. Danielius Serapinas ${ }^{a, b}$ y Prof. Asoc. Dra. Daiva Bartkeviciene
}

a. Departamento de Medicina Familiar, Academia Médica, Universidad Lituana de Ciencias de la Salud, Kaunas, Lituania.

b. Universidad Mykolas Romeris, Vilna, Lituania.

c. Departamento de Obstetricia y Ginecología, Facultad de Medicina, Universidad de Vilna, Vilna, Lituania.

Correspondencia:

Dr. Danielius Serapinas: dserapinas@gmail.com

Financiamiento: Ninguno.

Conflicto de intereses: Ninguno que declarar.

Recibido: 9-9-2018

Aceptado: 4-7-2019

\section{RESUMEN}

Objetivo. Investigar las tasas de trastornos respiratorios y del sueño en los niños cuyas madres se sometieron a una amniocentesis.

Materiales y métodos. Se incluyó a niños cuyas madres se sometieron a una amniocentesis en el segundo trimestre (entre las 16 y las 20 semanas) y otros sin procedimiento invasivo (controles).

Resultados. Se anallizó a 50 niños en el grupo de amniocentesis y a 47 controles. Hubo mayor incidencia de trastornos del sueño en el grupo de amniocentesis: 30 casos $(60 \%)$ frente a 11 controles $(23,4 \%)(p=0,001)$. En el grupo de amniocentesis, 7 niños (14\%) tenían asma; en el grupo de referencia, 1 niño $(2,1 \%)(p=0,032)$.

Conclusión. Podría haber una asociación entre la amniocentesis en el segundo trimestre, el asma y los trastornos del sueño en los niños. Se requieren estudios futuros y analizar los efectos a largo plazo de las pruebas invasivas.

Palabras clave: amniocentesis, consentimiento informado, enfermedades respiratorias, trastornos del sueño, niño. 